\title{
Valence-band density of states and surface electron accumulation in epitaxial $\mathrm{SnO}_{2}$ films
}

\author{
S. K. Vasheghani Farahani, ${ }^{1}$ T. D. Veal, ${ }^{2}$ J. J. Mudd, ${ }^{1}$ D. O. Scanlon, ${ }^{3,4}$ G. W. Watson, ${ }^{5}$ O. Bierwagen, ${ }^{6,7}$ M. E. White, ${ }^{6}$ \\ J. S. Speck, ${ }^{6}$ and C. F. McConville ${ }^{1, *}$ \\ ${ }^{1}$ Department of Physics, University of Warwick, Coventry CV4 7AL, United Kingdom \\ ${ }^{2}$ Stephenson Institute for Renewable Energy and Department of Physics, School of Physical Sciences, University of Liverpool, \\ Liverpool L69 7ZF, United Kingdom \\ ${ }^{3}$ Kathleen Lonsdale Materials Chemistry, Department of Chemistry, University College London, London WC1H OAJ, United Kingdom \\ ${ }^{4}$ Diamond Light Source Ltd., Diamond House, Harwell Science and Innovation Campus, Didcot, Oxfordshire OX11 ODE, United Kingdom \\ ${ }^{5}$ School of Chemistry and CRANN, Trinity College Dublin, Dublin 2, Ireland \\ ${ }^{6}$ Materials Department, University of California, Santa Barbara, California 93106-5050, USA \\ ${ }^{7}$ Paul-Drude-Institute für Festkörperelektronic, Hausvogteiplatz 5-7, D-10117 Berlin, Germany \\ (Received 25 June 2014; revised manuscript received 20 August 2014; published 8 October 2014)
}

\begin{abstract}
The surface band bending and electronic properties of $\mathrm{SnO}_{2}(101)$ films grown on $r$-sapphire by plasma-assisted molecular beam epitaxy have been studied by Fourier-transform infrared spectroscopy (FTIR), x-ray photoemission spectroscopy (XPS), Hall effect, and electrochemical capacitance-voltage measurements. The XPS results were correlated with density functional theory calculation of the partial density of states in the valence-band and semicore levels. Good agreement was found between theory and experiment with a small offset of the $\mathrm{Sn} 4 d$ levels. Homogeneous $\mathrm{Sb}$-doped $\mathrm{SnO}_{2}$ films allowed for the calculation of the bulk Fermi level with respect to the conduction-band minimum within the $\boldsymbol{k} \cdot \boldsymbol{p}$ carrier statistics model. The band bending and carrier concentration as a function of depth were obtained from the capacitance-voltage characteristics and model space charge calculations of the Mott-Schottky plots at the surface of Sb-doped $\mathrm{SnO}_{2}$ films. It was quantitatively demonstrated that $\mathrm{SnO}_{2}$ films have downward band bending and surface electron accumulation. The surface band bending, unoccupied donor surface-state density, and width of the accumulation region all decrease with increasing Sb concentration.
\end{abstract}

DOI: 10.1103/PhysRevB.90.155413

PACS number(s): 68.47.Gh, 79.60.Dp

\section{INTRODUCTION}

In materials with crystal structures, the perfect periodicity is broken at the surface, which introduces surface states with complex Bloch wave vectors. This means that surface states are evanescent and localized within the surface layer of the material (they decay exponentially into the vacuum and into the bulk). The generation of surface states is similar at a metal/semiconductor interface at which the wave functions of the metal can tunnel into the semiconductor and form states in the band gap [1]. At the free surface of an $n$-type semiconductor, donor (acceptor) defects and impurities are ionized when the respective surface states are unoccupied (occupied), which results in a downward (upward) band bending in the case of electron accumulation (depletion).

$\mathrm{SnO}_{2}$ is a transparent conducting oxide (TCO) well known in gas sensing [2,3] for its surface sensitivity and conductivity variations upon gas exposure. However, very little is known about the surface electronic properties of $\mathrm{SnO}_{2}$ films, because until recently high-quality thin films were not available. Highquality single-crystal $\mathrm{SnO}_{2}$ holds promising properties for electrical contact applications such as in transparent transistors [4,5]. Among the III-V semiconductors, InAs [6] and InN [7] are known to have surface electron accumulation layers. Most

\footnotetext{
*c.f.mcconville@warwick.ac.uk

Published by the American Physical Society under the terms of the Creative Commons Attribution 3.0 License. Further distribution of this work must maintain attribution to the author(s) and the published article's title, journal citation, and DOI.
}

binary TCOs that have attracted intensive research into their optical and electronic properties, namely, $\mathrm{In}_{2} \mathrm{O}_{3}[8,9]$, $\mathrm{CdO}$ [10-14], $\mathrm{ZnO}[11,15,16]$, and $\mathrm{SnO}_{2}$ [17-19], have all been shown to exhibit surface electron accumulation. This follows from the fact that the charge neutrality level (CNL), defined as the boundary energy at which defect states change from donor type to acceptor type with reference to the Fermi level, lies above the conduction-band minimum (CBM) in these materials. As a result, even high-work-function metals on $n$-type $\mathrm{SnO}_{2}$ form Schottky contacts only after an oxygenplasma treatment leading to the depletion of the surface electron accumulation [20,21]. For sensing and optoelectronic applications, the surface band bending and space charge properties are important and warrant investigation.

Over the past two decades density functional theory (DFT) calculations using hybrid functionals have been shown to produce improved descriptions of structure, band gap, and defect properties of a range of binary, ternary, and quaternary oxide semiconductors [22-30]. In spite of some density functional theory calculations of the density of states (DOS) in $\mathrm{SnO}_{2}$ [24,31] with comparisons to photoemission spectra [21], no detailed work has been accomplished to correlate the two through the application of photoionization cross sections on the calculated partial density of states (PDOS). Concerning the surface Fermi level pinning in $\mathrm{SnO}_{2}$ thin films, Nagata et al. [17] have investigated the binding energy of the core Sn $3 d$ electrons by $\mathrm{Al} \mathrm{K} \alpha$ and hard $\mathrm{x}$-ray photoemission spectroscopy (XPS) and schematically illustrate a downward band bending at the surface of the films. A lack of a quantitative study on the surface band bending and electron accumulation as a function of depth is also perceived in epitaxial $\mathrm{SnO}_{2}$ films. 
In this paper, experimental characterization techniques together with DFT calculations and theoretical modeling of the band extrema and electron distribution in the bulk and at the surface of $\mathrm{SnO}_{2}(101) / r$-sapphire have been employed to deduce the surface electronic properties of epitaxial $\mathrm{SnO}_{2}$ (101) films. We show that the bulk physical quantities necessary for surface electronic calculations are not attainable for unintentionally doped (UID) epitaxial $\mathrm{SnO}_{2}$ due to the background donor density and carrier concentration gradients throughout the films. By modeling the Mott-Schottky plots, we quantitatively present the surface band bending as a function of depth and the corresponding electron accumulation profiles in Sb-doped $\mathrm{SnO}_{2}(101)$ films. Variation of the unoccupied donor surface-state density is obtained as a function of Sb-doping level.

\section{EXPERIMENTAL AND THEORETICAL METHODS}

The samples used in this study were UID and antimony(Sb-) doped $\mathrm{SnO}_{2}$ (101) films grown on $r$-sapphire as the substrate by plasma-assisted molecular beam epitaxy (PAMBE). Standard Knudsen effusion cells were used to evaporate $\mathrm{Sn}$ (99.9999\% pure) and Sb (99.999\% pure), and a Veeco UNIBulb radio-frequency plasma source to supply monatomic oxygen. The $\mathrm{Sn}$ and maximum $\mathrm{Sb}$ source cell temperatures were 1270 and $600{ }^{\circ} \mathrm{C}$, respectively. The substrate temperature for both sets of samples was maintained at $700^{\circ} \mathrm{C}$. Further description of the growth process can be found elsewhere $[32,33]$. The UID samples were grown in a range of thicknesses between 26 and $1505 \mathrm{~nm}$. The Sb-doped samples were grown at a fixed thickness of around $550 \mathrm{~nm}$ on a UID $\mathrm{SnO}_{2}$ film of thickness $\approx 480 \mathrm{~nm}$ as an intermediate layer. Secondary ion mass spectrometry (SIMS) was used to determine the $\mathrm{Sb}$ concentration in the samples which ranged between $9.8 \times 10^{17}$ and $3.5 \times 10^{19} \mathrm{~cm}^{-3}[33]$.

Mid-infrared (MIR) reflectance and Hall effect measurements were carried out to determine the thickness, carrier concentration, plasmon energy, and mobility of the films. XPS spectra were collected under ultrahigh vacuum $(3 \times$ $10^{-11}$ mbar) using a monochromated $\mathrm{Al} \mathrm{K} \alpha$ x-ray beam $(h v=$ $1486.6 \mathrm{eV}$ ) to investigate the valence-band and semicore level energies at the surface of the $\mathrm{SnO}_{2}$ films. The emitted photoelectrons were detected in normal emission using an Omicron SPHERA electrostatic hemispherical deflection analyzer with a mean radius of $125 \mathrm{~mm}$. The overall instrumental energy resolution was $0.4 \mathrm{eV}$. The spectrometer binding energy scale was calibrated with reference to sputtered polycrystalline silver. Electrochemical capacitance-voltage (ECV) measurements were performed using a WEP/Dage Wafer Profiler CVP21 system. GaIn eutectic was used to ensure Ohmic contacts between the sample and the terminals followed by $I-V$ measurements across the films. The electrolyte used for the $\mathrm{SnO}_{2}$ samples was 1 molar $\mathrm{SnCl}_{4}$ diluted in deionized water. The voltage is measured with respect to the saturated calomel electrode (SCE) placed into the electrolyte. All measurements were performed at room temperature.

DFT calculations were perfomed using the VASP code $[34,35]$ in which a plane-wave basis set is used to describe the valence electronic states. The Perdew-Burke-Emzerhof [36] (PBE) gradient-corrected functional was used to treat the exchange and correlation. Interaction between the cores (Sn:[Kr] and O:[He]) and the valence electrons was described using the projector-augmented wave [37,38] method, with $\mathrm{Sn} 4 d$ states explicitly included in the valence. We have employed the hybrid density functional developed by Adame and Barone (PBE0) (Ref. [39]) as implemented in the VASP code [40] to obtain the band structure and PDOS of the core and valence-band electrons. In the PBE0 formalism, a value $(\alpha)$ of exact nonlocal exchange of $25 \%$ is added to the PBE formalism. Structural optimizations of bulk $\mathrm{SnO}_{2}$ were performed using PBE0 at a series of volumes in order to calculate the equilibrium lattice parameters. In each case the atomic positions, lattice vector, and cell angle were allowed to relax, while the total volume was held constant. The resulting energy volume curves were fitted to the Murnaghan equation of state to obtain the equilibrium bulk cell volume [41]. Convergence with respect to $k$-point sampling and planewave energy cutoff were checked, with a cutoff of $400 \mathrm{eV}$ and a $k$-point sampling of $4 \times 4 \times 6$ found to be sufficient. Calculations were deemed to be converged when the forces on all the atoms were less than $0.01 \mathrm{eV} / \AA$.

A two-band $\boldsymbol{k} \cdot \boldsymbol{p}$ model was used for carrier statistics calculations to evaluate the bulk Fermi level of the films. The surface band bending and carrier concentration profiles as functions of depth were computed by solving the Poisson equation. The modified Thomas-Fermi approximation (Refs. [42-44]) was employed to account for the boundary conditions at the surface of the films. The initial input parameters for this calculation were the energy separation of the Fermi level from the valence-band maximum (VBM) at the surface (determined by XPS) and to the CBM in the bulk (using the Hall effect data).

\section{RESULTS AND DISCUSSION}

\section{A. Infrared reflectance and Hall effect}

The thicknesses $d$ of the UID and Sb-doped films have been determined by simulating the measured $s$-polarized reflectance spectra. The electric field of radiation was oriented in order to satisfy the condition $\boldsymbol{E} \perp \boldsymbol{c}$, where $\boldsymbol{c}$ is the principal axis. The reflectance was measured at an incident light angle of $56^{\circ}$ with respect to the surface normal and simulated assuming a three-layer (vacuum/UID $\mathrm{SnO}_{2} /$ sapphire) and a fourlayer (vacuum/Sb-doped $\mathrm{SnO}_{2} / \mathrm{UID} \mathrm{SnO}_{2} /$ sapphire) stratified medium for the UID and Sb-doped $\mathrm{SnO}_{2}$ films, respectively. The plots are shown in Fig. 1 for four samples. The carrier concentration of the films $n_{\mathrm{b}}$ was calculated using the sheet carrier concentration $n_{\mathrm{s}}$ from the Hall effect measurements according to $n_{\mathrm{b}}=n_{\mathrm{s}} / d$. Since the $\mathrm{Sb}$-doped $\mathrm{SnO}_{2}$ films were grown on a semiconducting intermediate layer, the parallel conduction method has been used to calculate the carrier concentration and mobility of the doped samples [46]. The values for the UID (Sb-doped $\mathrm{SnO}_{2}$ ) films range between $n_{\mathrm{b}}=3.1 \times 10^{17}$ and $n_{\mathrm{b}}=2.4 \times 10^{18} \mathrm{~cm}^{-3}\left(n_{\mathrm{b}}=1.1 \times 10^{18}\right.$ and $\left.n_{\mathrm{b}}=2.6 \times 10^{19} \mathrm{~cm}^{-3}\right)$. The corresponding Sb-doping levels for each carrier concentration are given in Table I. The average plasmon excitation energies of the films were obtained in the modeling as well as by carrier statistics calculations using the pertinent formula:

$$
E_{\mathrm{p}}=\hbar\left(\frac{n e^{2}}{m^{*} \epsilon_{0} \epsilon(\infty)}\right)^{1 / 2},
$$




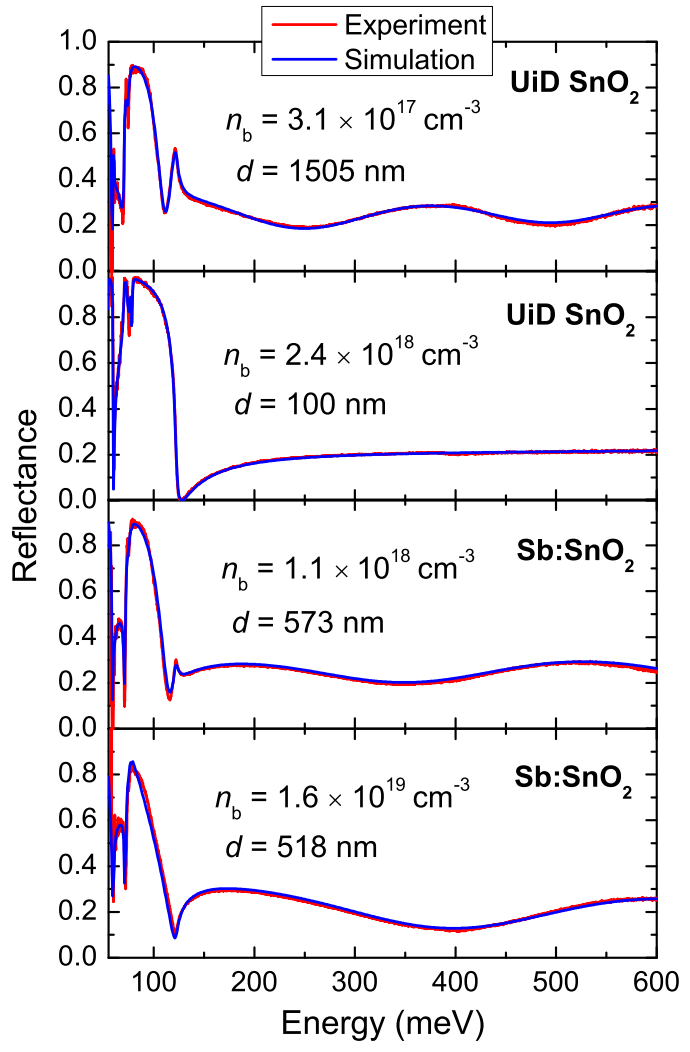

FIG. 1. (Color online) MIR reflectance data and simulation for two UID and Sb-doped $\mathrm{SnO}_{2}$ samples between 50 and $600 \mathrm{meV}$. The UID samples have different thicknesses with varying carrier concentrations, indicative of the presence of charged dislocations in the films [45]. The Sb-doped $\mathrm{SnO}_{2}$ films have close thicknesses with different $\mathrm{Sb}$ concentrations. $n_{\mathrm{b}}=1.1 \times 10^{18}$ and $1.6 \times 10^{19} \mathrm{~cm}^{-3}$ correspond to $\mathrm{Sb}$ concentrations of $n_{\mathrm{Sb}}=9.8 \times 10^{17}$ and $2.1 \times$ $10^{19} \mathrm{~cm}^{-3}$, respectively.

where $n$ is the electron concentration, $m^{*}$ is the effective mass, and $\epsilon_{0}[\epsilon(\infty)]$ is the static (high-frequency) dielectric constant.

\section{B. Electronic density of states of the valence-band and semicore levels}

DFT calculations were performed for undoped $\mathrm{SnO}_{2}$ to obtain the PDOS in the valence-band and core levels. Figures 2(a)-2(c) shows the DFT density of states of the Sn and $\mathrm{O}$ orbitals in $\mathrm{SnO}_{2}$ for the valence-band and the shallow and deeper semicore levels. The valence band is dominated by the $\mathrm{O} 2 p$ orbitals while the shallow and deeper semicore levels are predominantly $\mathrm{O} 2 s$ and $\mathrm{Sn} 4 d$, respectively. The binding energy spectra of electrons below the VBM were acquired

TABLE I. The bulk electron and corresponding Sb concentrations for each sample, all having the same thickness.

\begin{tabular}{cc}
\hline \hline Carrier concentration $\left(\mathrm{cm}^{-3}\right)$ & Sb concentration $\left(\mathrm{cm}^{-3}\right)$ \\
\hline $1.1 \times 10^{18}$ & $9.8 \times 10^{17}$ \\
$3.4 \times 10^{18}$ & $4.5 \times 10^{18}$ \\
$1.6 \times 10^{19}$ & $2.1 \times 10^{19}$ \\
$2.6 \times 10^{19}$ & $3.5 \times 10^{19}$ \\
\hline
\end{tabular}

by XPS measurements. The zero of the binding energy scale was set at the Fermi level. Corresponding to the X-ray energy $h v=1486.6 \mathrm{eV}$, the one-electron angular-corrected photoionization cross sections, taking into account the orbital dipole asymmetry parameters, have been applied $[47,48]$ to the calculated PDOS plots in Figs. 2(a)-2(c). The results are presented in Figs. 2(d)-2(f). Due to the high relative photoionization cross section of $\mathrm{Sn} 4 d$, it is observed that photoemitted electrons from the VBM and deeper semicore levels would originate primarily from $\mathrm{Sn} 4 d$ orbitals.

The photoionization cross section corrected DFT DOS are correlated with the XPS binding energy spectra of the films in Fig. 3. The weighted DFT PDOS were summed and convoluted with $0.1 \mathrm{eV}$ Lorentzian broadening and $0.4 \mathrm{eV}$ Gaussian broadening to account for the lifetime and instrumental broadening, respectively. As the experimental results shown are for a sample with $n_{\mathrm{b}}=1.6 \times 10^{19} \mathrm{~cm}^{-3}$ with the Fermi level lying $0.075 \mathrm{eV}$ above the conduction-band minimum, the calculated DFT curves were shifted to fit the Shirley-background-subtracted [49] photoemission spectra at the VBM, because in the DFT calculations, the VBM is chosen to be the zero of the energy scale by convention.

The theory and experiment are in excellent agreement in shape and width across the VB as can be seen in Fig. 3(a). At the semicore levels in Fig. 3(b), a broader asymmetric line shape on the high-binding-energy side of the peaks is observed in photoemission compared to theory. This broadening is attributed to final-state effects manifested as satellite peaks within the main semicore level peak due to inelastic plasmon losses in the accumulation region [50,51]. More broadening is observed in the Sb-doped film with a higher plasmon energy and Hall carrier concentration relative to the UID film. The energy separation on the high-binding-energy side of the peaks, $\delta E \approx 135 \mathrm{meV}$, is found to be almost identical to the difference in the average plasmon energy in the films, $\delta E_{\mathrm{p}} \approx 130 \mathrm{meV}$, determined from MIR reflectance simulations as well as carrier statistics calculations. This supports the assignment of this broadened feature to plasmon excitations of free electrons in the surface electron accumulation layer, and changes to the surface space charge with Sb doping.

An energy separation of $\Delta E \approx 1.5 \mathrm{eV}$ is observed between theory and experiment for the deeper semicore levels $(E>$ $24 \mathrm{eV}$ ) where the photoemission is dominated by the $\mathrm{Sn} 4 d$ orbitals due to their high photoionization cross section [see Fig. 2(d)]. This slight discrepancy can be attributed to an incorrect cancellation of the self-interaction error in the hybrid DFT approach used. Unusually, this problem cannot be solved by going to higher levels of theoretical modelling such as the GW approximation [52]. To date the only possible correction has been the application of an empirical nonlocal external potential for the $d$ states, which is fitted to reproduce experimental $d$-band positions $[53,54]$. In this study, we choose not to artificially augment our calculations with any external potentials, as it will have no bearing on the generality of our results.

\section{Surface band bending and the bulk Fermi level}

To obtain the magnitude of surface band bending for each sample, the binding energy $(\xi)$, defined as the energy separation between the VBM at the surface and the Fermi level, was determined from the XPS spectra by the extrapolation of 

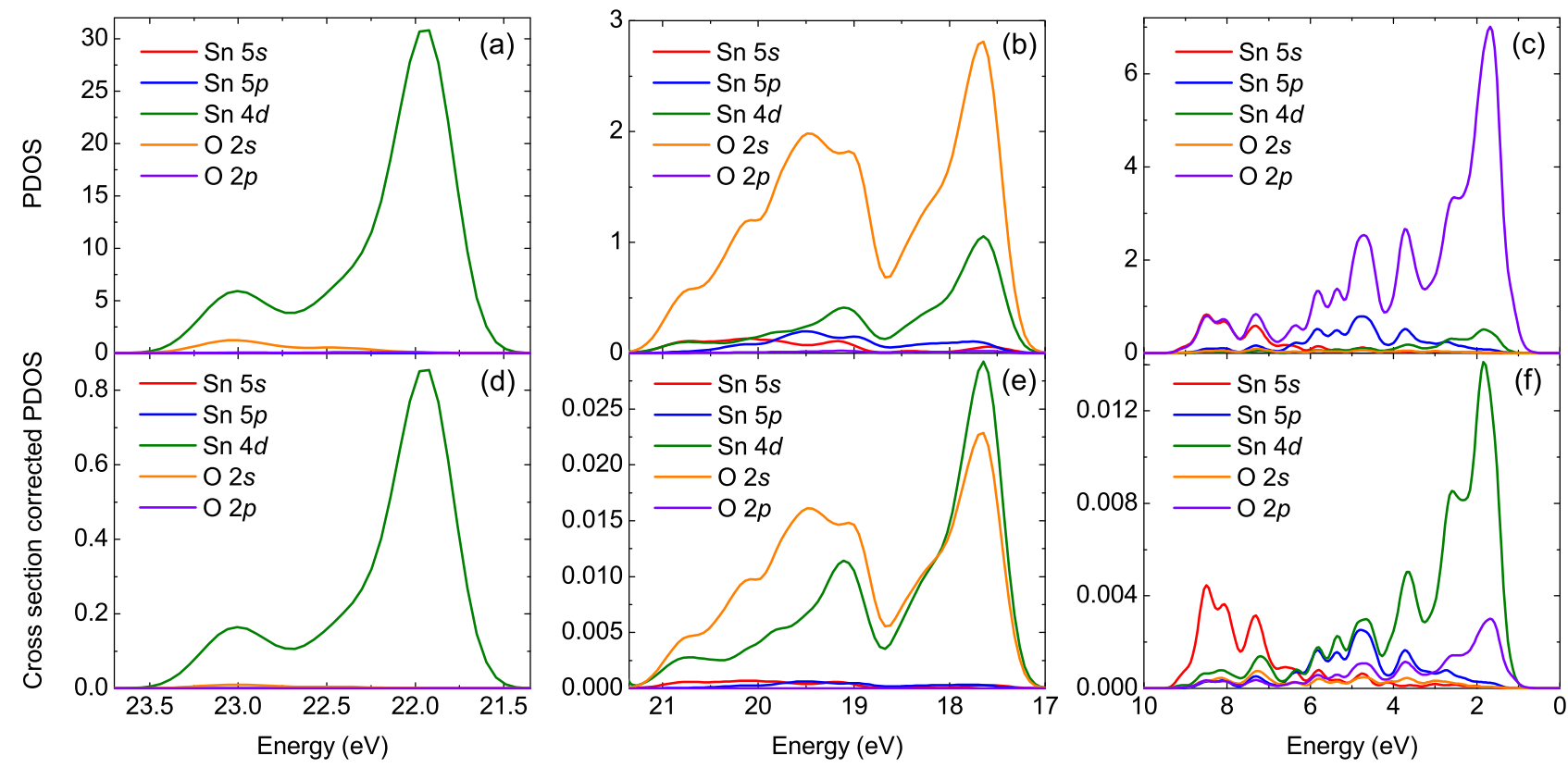

FIG. 2. (Color online) Density functional theory calculations of the PDOS of the VB and semicore levels in $\mathrm{SnO}_{2}$. The VBM is placed at $0 \mathrm{eV}$. (a)-(c) The valence band, lying within 0 and $10 \mathrm{eV}$, is dominated by $\mathrm{O} 2 p$ orbitals for energies below $7 \mathrm{eV}$, and constructed equally of $\mathrm{O} 2 p$ and $\mathrm{Sn} 5 s$ orbitals at the underlying energy levels. At the core levels, the density of $\mathrm{O} 2 s$ states is highest in the shallower region while Sn $4 d$ dominates at deeper levels. (d)-(f) Spectral modulation of the calculated PDOS in (a)-(c) by applying the one-electron and angular cross sections to the corresponding orbitals. It is observed that in spite of the dominance of O $2 p$ orbitals at the VBM, electrons would be photoemitted mainly from Sn $4 d$ orbitals because of their higher photoionization cross section. The same effect is observed for the entire range of deeper semicore levels as the region is dominated by $\mathrm{Sn} 4 d$.

the sharp linear onset of the intensity onto the energy axis. This is shown in Fig. 3(a). The surface band bending $V_{\mathrm{bb}}$ was evaluated using the equation

$$
V_{\mathrm{bb}}=\xi-E_{\mathrm{g}} \pm E_{\mathrm{F}},
$$

where $E_{\mathrm{g}}=3.5 \mathrm{eV}$ is the band gap of $\mathrm{SnO}_{2}[55,56] . E_{\mathrm{F}}$ is the bulk Fermi level calculated with respect to the CBM within the $\boldsymbol{k} \cdot \boldsymbol{p}$ model using carrier concentrations obtained from Hall effect measurements; the plus (minus) sign for $E_{\mathrm{F}}$ corresponds to $E_{\mathrm{F}}$ located below (above) the CBM. The sur face band bending and carrier concentration for both the UID and Sb-doped samples are shown in Table II. Since the band bending is determined relative to the band extrema in the bulk, the negative sign signifies the accumulation of the electrons at the surface where there exists a downward band bending.

TABLE II. Surface band bending $V_{\mathrm{bb}}(\mathrm{eV})$ and corresponding bulk carrier concentration from Hall effect measurements, $n_{\mathrm{b}}\left(\mathrm{cm}^{-3}\right)$, of the UID and Sb-doped $\mathrm{SnO}_{2}$ films.

\begin{tabular}{ccccc}
\hline \hline \multicolumn{2}{c}{$\mathrm{UID} \mathrm{SnO}_{2}$} & & \multicolumn{2}{c}{ Sb-doped $\mathrm{SnO}_{2}$} \\
\cline { 5 - 5 }$V_{\mathrm{bb}}$ & $n_{\mathrm{b}}$ & & $V_{\mathrm{bb}}$ & $n_{\mathrm{b}}$ \\
\hline-0.263 & $3.1 \times 10^{17}$ & & -0.327 & $1.1 \times 10^{18}$ \\
-0.258 & $3.8 \times 10^{17}$ & & -0.293 & $3.4 \times 10^{18}$ \\
-0.231 & $1.0 \times 10^{18}$ & & -0.226 & $1.6 \times 10^{19}$ \\
-0.220 & $1.5 \times 10^{18}$ & & -0.192 & $2.6 \times 10^{19}$ \\
-0.206 & $2.4 \times 10^{18}$ & & & \\
\hline \hline
\end{tabular}

Concerning the UID samples, there is a nonuniform distribution of carrier concentration for regions below the surface layer towards the interface due to the depth-dependent charged-dislocation density [45]. This implies that the separation of the CBM from the Fermi level is not constant in the bulk due to variations in background donor density. Hence, the surface band bending could not be calculated for the UID $\mathrm{SnO}_{2}$ films grown on $r$-sapphire. For the Sb-doped films, the electron concentration increases with $\mathrm{Sb}$ concentration, indicating that $\mathrm{Sb}$ acts as a donor in $\mathrm{SnO}_{2}$. These films were grown to a thickness of $550 \mathrm{~nm}$ on an intermediate layer of UID $\mathrm{SnO}_{2}$ with a relatively high thickness of $\approx 480 \mathrm{~nm}$. Therefore, the lattice mismatch has significantly decreased, leading to a considerable reduction in the overall dislocation density. This is confirmed by a significant decrease in the dislocation scattering as compared with the UID films in Fig. 4. Since charged-dislocation scattering is the dominant scattering mechanism at higher carrier concentrations [45], the electron mobility difference between the UID and Sb-doped $\mathrm{SnO}_{2}$ is expected to increase with carrier concentration as is seen in Fig. 4. This implies that the energy of the CBM in the bulk is constant with respect to the Fermi level, resulting from a homogeneous distribution of $\mathrm{Sb}$ donors and charge carriers in Sb-doped $\mathrm{SnO}_{2}$ films. The issue with the UID samples is further highlighted in Table II by comparing the degree of calculated band bending [Eq. (2)] and the corresponding carrier concentration between the UID and Sb-doped $\mathrm{SnO}_{2}$ films; the band bending is calculated using the Hall effect which "averages" over the entire film. This is considered in conjunction with Fig. 5 which illustrates the 

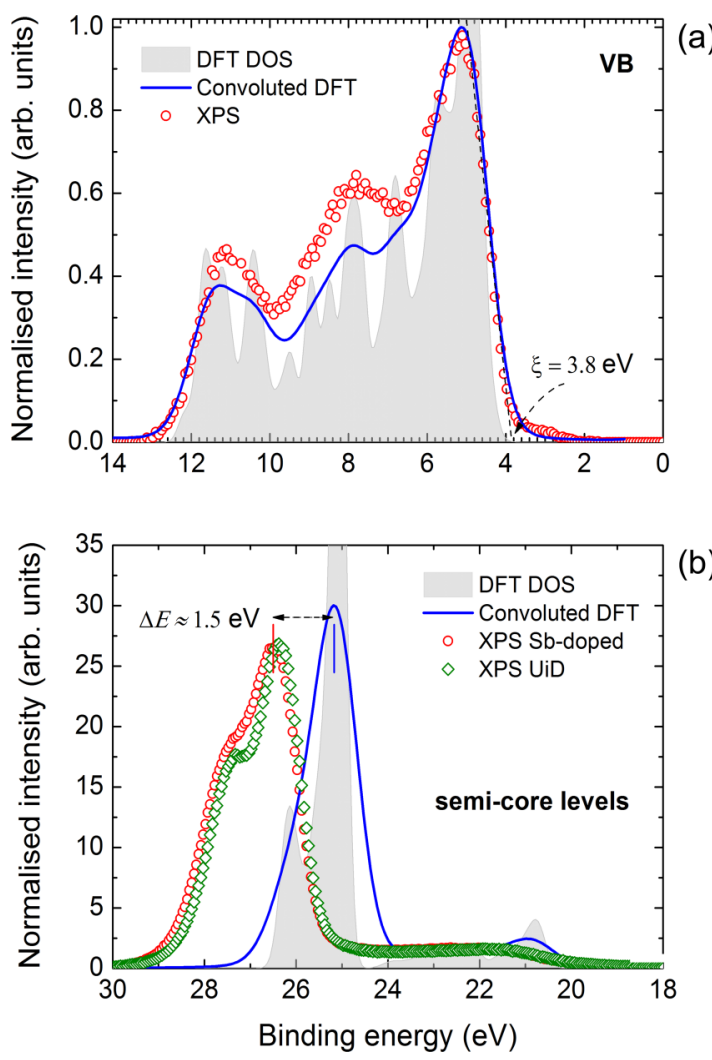

FIG. 3. (Color online) Comparison of the cross section weighted DFT PDOS with the Shirley-background-subtracted XPS spectra for (a) valence band and (b) semicore levels in $\mathrm{SnO}_{2}$. The graphs are normalized to the maximum of the valence-band peak. The gray-shaded area plots are the summed-up photoionization cross section weighted DFT PDOS. The weighted DOS has been broadened with a Lorentzian function followed by a Gaussian to correlate with experiment. The XPS spectra (red circles) pertain to an Sb-doped film with a bulk carrier concentration $n_{\mathrm{b}}=1.6 \times 10^{19} \mathrm{~cm}^{-3}$. Hence, the DFT DOS spectrum is shifted to fit the experimental VBM. At the semicore levels in (b), the line shape of a UID film with a lower Hall-averaged carrier concentration is less broadened on the high-binding-energy side.

band bending variations with respect to the separation of the bulk Fermi level from the CNL. The surface band bending is expected to decrease as the bulk carrier concentration increases which moves the bulk Fermi level closer to the CNL, but higher band bending is observed for the Sb-doped films that have the same carrier concentration as the UID samples.

\section{ECV measurements and space charge calculations}

Electron accumulation layers at the surface of semiconductors lead to Ohmic metal contacts, preventing the formation of the rectifying Schottky contacts required for conventional $C-V$ measurements. Here, ECV measurements were used instead as the Helmholtz double layer at the electrolyte/semiconductor interface behaves as a "Schottky-like" contact. The surface band bending and electron concentration as a function of depth were calculated for $\mathrm{Sb}$-doped $\mathrm{SnO}_{2}$ films by measuring the $C-V$ characteristics and simulation of the Mott-Schottky plots

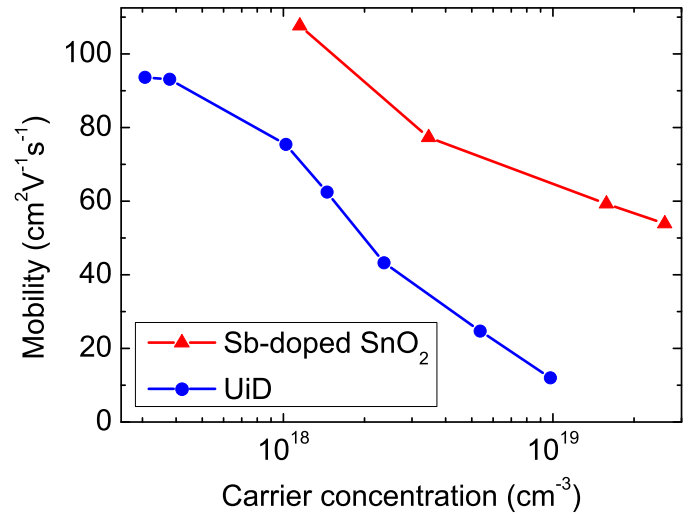

FIG. 4. (Color online) Comparison between the UID and Sbdoped $\mathrm{SnO}_{2}$ films in respect of carrier mobility as a function of carrier concentration. Lines are guides to the eye. The UID $\mathrm{SnO}_{2}$ films were grown directly on $r$-sapphire which showed a high density of charged dislocations, while the Sb-doped films were grown on an intermediate UID $\mathrm{SnO}_{2}$ layer. It is apparent that the mobilities in the Sb-doped films are much higher than those in the UID films in terms of carrier concentration. This clearly illustrates the significant reduction in dislocation scattering in the Sb-doped films.

$\left(1 / C^{2}\right.$ as a function of the applied bias). The Poisson equation was solved assuming a negligible amount of acceptor defects or impurities:

$$
\frac{d^{2} V}{d z^{2}}=-\frac{e}{\epsilon_{\mathrm{s}} \epsilon_{0}}\left[N_{\mathrm{D}}^{+}-n(z)\right]
$$

where the boundary conditions were applied according to the expressions

$$
z \rightarrow \infty \Longrightarrow V(z) \rightarrow 0,\left.\quad \frac{d V}{d z}\right|_{z=0}=\frac{e}{\epsilon_{\mathrm{s}} \epsilon_{0}} n_{\mathrm{ss}},
$$

where $n_{\mathrm{ss}}$ is the surface sheet density of the semiconductor. Due to the optical and electronic anisotropy of $\mathrm{SnO}_{2}$, the

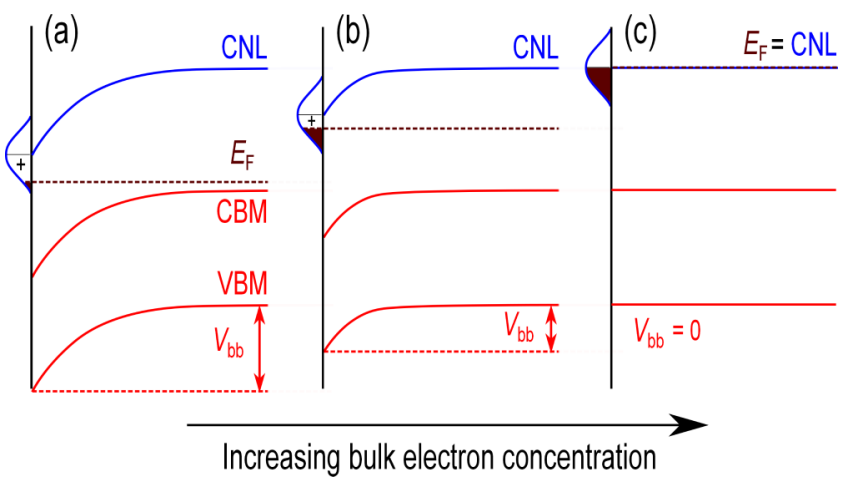

FIG. 5. (Color online) Schematic representation of the decrease in downward band bending, $V_{\mathrm{bb}}$, as the bulk Fermi level moves up towards the CNL (a)-(c). As the carrier concentration increases due to $n$-type doping of the bulk, a reduction in the density of unoccupied surface states is observed until the bulk and surface Fermi levels stabilize at the CNL (c). 


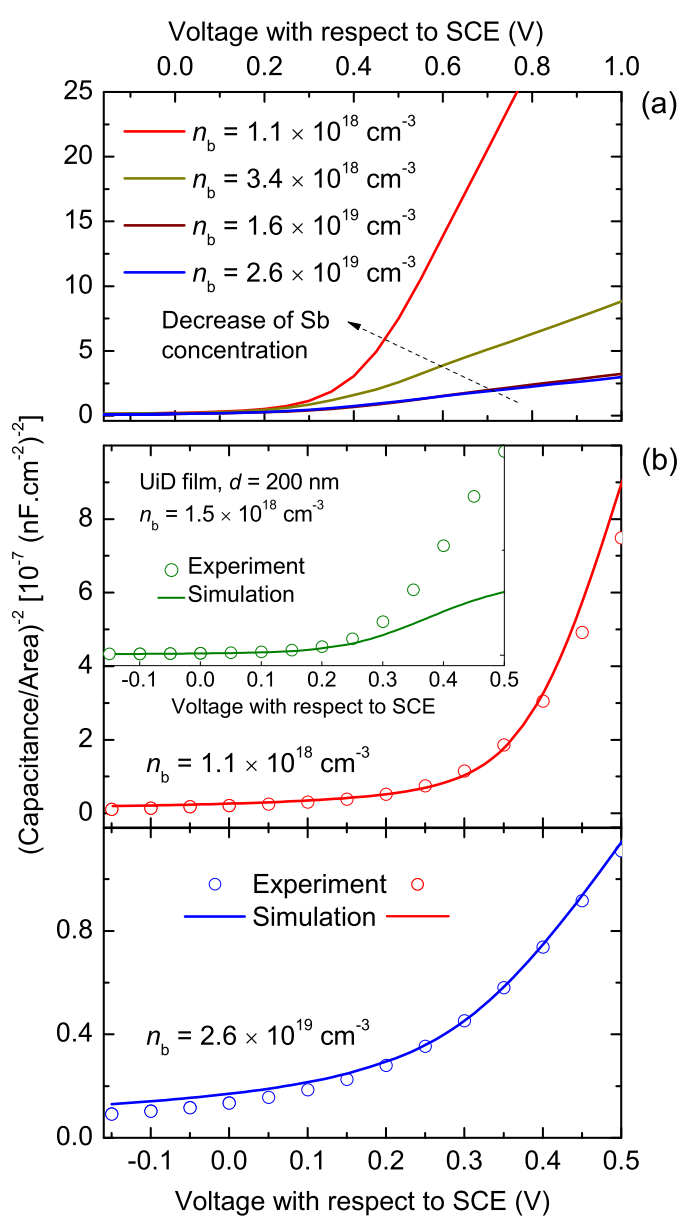

FIG. 6. (Color online) ECV data and simulation of the MottSchottky curves in Sb-doped $\mathrm{SnO}_{2}$ films. (a) Experimental data of the four investigated samples. The magnitude of $1 / C^{2}$ decreases with bulk carrier concentration of the films, implying that the electron concentration at the surface increases with $\mathrm{Sb}$ concentration. The shallow gradient region at lower bias for each sample indicates the depletion of the surface electron accumulation and the steeper region at higher bias is indicative of lower net space charge in the bulk. (b) shows the Mott-Schottky plots with corresponding model curves of the samples with the lowest and highest bulk carrier concentrations, respectively. The model curve of a UID sample with a thickness of $200 \mathrm{~nm}$ is compared to the experiment in the inset of (b). It is apparent that the data could not be reproduced since the donor density in the bulk is nonuniform.

band edge effective mass and the dielectric constants were calculated via

$$
m_{0}^{*}=\left(m_{0, \perp}^{* 2} m_{0, \|}^{*}\right)^{1 / 3}, \quad \epsilon=\frac{2 \times \epsilon_{\perp}+\epsilon_{\|}}{3} .
$$

$m_{0, \perp}^{*}\left(0.3 m_{0}\right), m_{0, \|}^{*}\left(0.23 m_{0}\right)[57,58], \epsilon_{\perp}(0)(13.5)$, and $\epsilon_{\|}(0)$ (9.58) (Ref. [59]) give an average band edge effective mass and static dielectric constant values of $0.27 m_{0}$ and 12.2, respectively.

The capacitance-voltage data in the form of Mott-Schottky plots for four $\mathrm{Sb}$-doped $\mathrm{SnO}_{2}$ films are shown in Fig. 6(a). The positive slope of the plots indicates the $n$-type character of the films. The magnitude of $1 / C^{2}$ decreases with bulk carrier concentration of the films, implying that the electron concentration at the surface increases with Sb concentration. The shallow gradient region at lower bias for each sample indicates the depletion of the surface electron accumulation and the steeper region at higher bias is indicative of lower net space charge in the bulk. The constant slopes in the Mott-Schottky plots which correspond to the bulk carrier density in each sample agree well with the bulk values derived from reflectance simulations and the Hall data for the Sb-doped films.

The Mott-Schottky plots and respective model curves of two Sb-doped samples with the lowest and highest bulk carrier concentrations are shown in Fig. 6(b). The model curves obtained by solving the Poisson equation assuming a uniform background of ionized donors bear out the homogeneity of the donor distribution near the surface. The simulation was performed after the evaluation of the bulk Fermi level by carrier statistics calculations within the two-band $\boldsymbol{k} \cdot \boldsymbol{p}$ model and obtaining the degree of surface band bending via Eq. (2) using the XPS results. As can be seen in the inset of Fig. 6(b), the modeling of the Mott-Schottky plots for UID films showing a lower linear slope was not possible. This is because of the nonuniform charged-dislocation density as a function of depth, which results in a higher "bulk" carrier concentration and Fermi level (from the Hall effect data) than those in the immediate vicinity below the surface layer (obtained from the linear slope of the Mott-Schottky plots) - the correct value to be used in space charge calculations. This clearly substantiates the nonuniform density of background donors in the UID films discussed in the previous section.

Modeling of the Mott-Schottky curves enabled the calculation of the carrier concentration and band bending as a function of depth at the surface of the $\mathrm{Sb}$-doped $\mathrm{SnO}_{2}$ films. This is shown in Fig. 7 for the lowest- and highestSb-doped $\mathrm{SnO}_{2}$ films together with the position of the Fermi level. All the films show downward band bending and surface electron accumulation. As can be seen in Fig. 7(a), the surface band bending in the film with a lower bulk electron concentration and $\mathrm{Sb}$ doping level was larger and the corresponding surface layer wider compared to those in the film with a higher electron and $\mathrm{Sb}$ concentration. Accordingly, the deduced surface-state densities from the modeling show that the density of unoccupied donor surface states, $n_{\mathrm{ss}}$, decreases with $\mathrm{Sb}$ doping. The bulk Fermi level for the sample with the lowest $\mathrm{Sb}$ concentrations, namely, $9.8 \times 10^{17} \mathrm{~cm}^{-3}$, lies $0.027 \mathrm{eV}$ below the CBM in the band gap, being distant from the CNL of $\mathrm{SnO}_{2}$ which is at 0.5 $\mathrm{eV}$ above the CBM. A higher Sb concentration of $3.5 \times 10^{19}$ $\mathrm{cm}^{-3}$ moves the bulk Fermi level up into the conduction band, reaching $0.109 \mathrm{eV}$, reducing the band bending and surface-state density [60]. Figure 7(b) profiles the electron accumulation at the surface of the Sb-doped $\mathrm{SnO}_{2}$ films. The width of the surface accumulation layer decreases with the Fermi level. Hence, the variation in the density of surface-state charge is depicted as a function of Sb concentration in Fig. 8. The results in Fig. 8 quantitatively demonstrate the phenomenon schematically shown in Fig. 5. Since these unoccupied donor surface states lie above the pinned Fermi level, they become increasingly occupied, and therefore neutral, as the bulk Fermi level approaches the CNL due to increasing levels of $\mathrm{Sb}$ doping. 


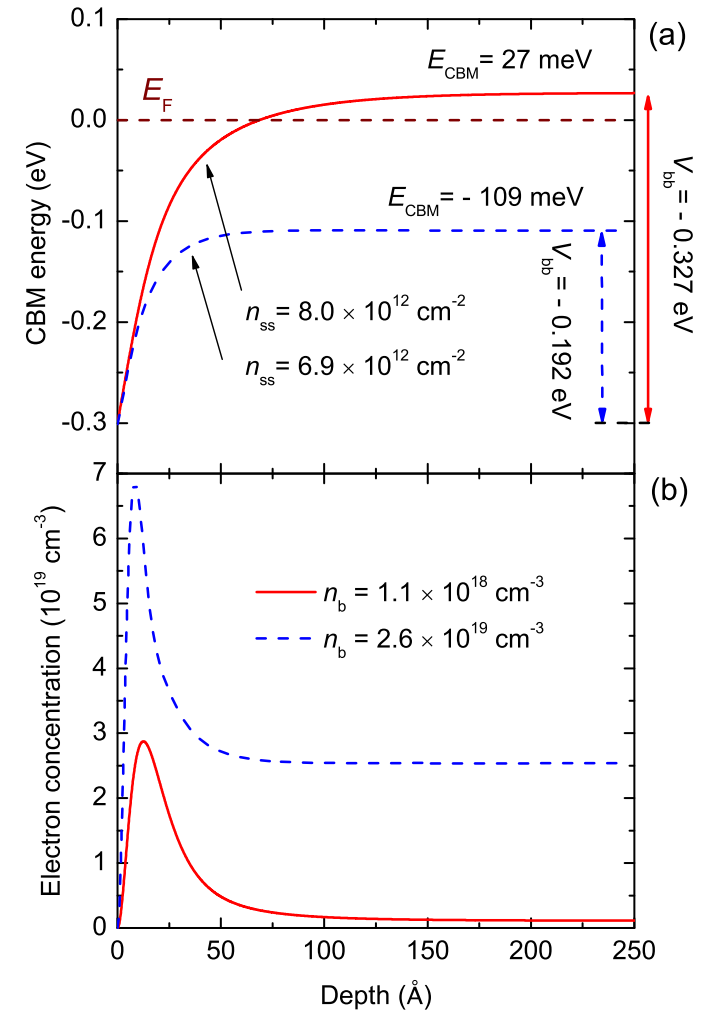

FIG. 7. (Color online) The calculated surface band bending $V_{\mathrm{bb}}$ and electron concentration $n$ as functions of depth, together with the corresponding surface-state density $n_{\mathrm{ss}}$ for two Sb-doped $\mathrm{SnO}_{2}$ samples. (a) shows $V_{\mathrm{bb}}$ as a function of depth for the films with the lowest and highest $\mathrm{Sb}$ concentrations corresponding to carrier concentrations $n_{\mathrm{b}}=1.1 \times 10^{18} \mathrm{~cm}^{-3}$ and $n_{\mathrm{b}}=2.6 \times 10^{19} \mathrm{~cm}^{-3}$, respectively. The corresponding charge profiles at the surface are plotted in (b). The horizontal dashed line is the location of the pinned Fermi level taken as the zero energy scale. The reduction in the surface band bending and accumulation layer width along with the increase in the surface electron concentration is apparent as the bulk Fermi level moves to higher energies in the conduction band. The density of unoccupied donor surface states decreases with Sb doping.

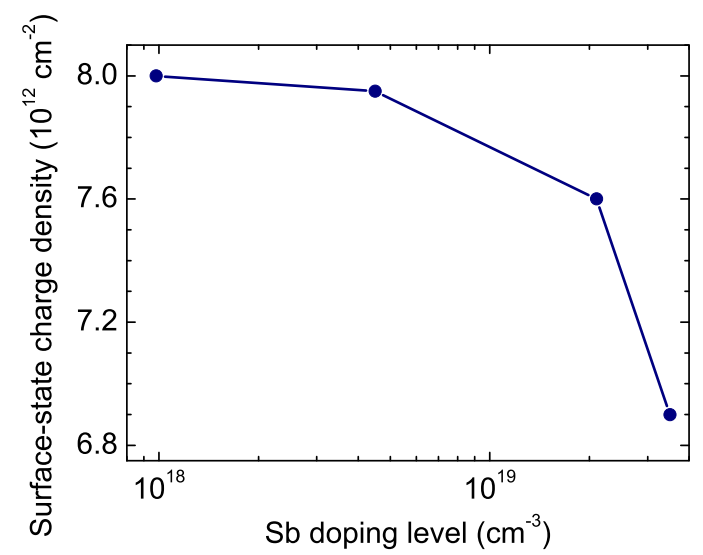

FIG. 8. (Color online) Unoccupied donor surface-state density, obtained from space charge calculations, decreases with $\mathrm{Sb}$ doping level. This points to the reduction of surface space charge and band bending to maintain charge neutrality at the surface, with the Fermi level rising further towards the CNL with Sb doping.

\section{SUMMARY AND CONCLUSIONS}

We present a detailed experimental and theoretical study of the surface electronic properties of high-quality $\mathrm{SnO}_{2}(101)$ films grown on $r$-sapphire by PAMBE. A combination of FTIR spectroscopy, Hall effect, XPS, and ECV measurements have all been used in conjunction with first-principles DFT, carrier statistics, and space charge calculations for quantitative analysis of the surface charge characteristics and band bending of epitaxial UID and Sb-doped $\mathrm{SnO}_{2}$ thin films.

The calculated DFT PDOS were correlated with XPS results by applying the one-electron angular photoionization cross section corrected orbitals in the valence-band and semicore levels and the results were shown to be in good agreement with experiment. By the alignment of the valenceband edge, the surface binding energy of the electrons was determined to be $3.8 \mathrm{eV}$ at the VBM. A discernible energy separation of $\Delta E \approx 1.5 \mathrm{eV}$ was observed between DFT and XPS results for the deeper semicore levels due to the underestimation of core $d$ levels in DFT calculations and their high photoionization cross section in XPS. The energy separation on the high-binding-energy side of the XPS peak of the semicore levels between the UID and Sb-doped samples is shown to be almost identical to their average plasmon energy difference obtained from both MIR reflectance simulations and carrier statistics calculations. This corroborates the model of inelastic energy losses of photoemitted electrons to conduction band plasmons in surface accumulation layers.

The UID $\mathrm{SnO}_{2}$ films contained a high density of charged dislocations originating at the interface and reducing towards the surface. Hence, due to the nonuniform background ionized donor density, a constant separation of the CBM from the bulk Fermi level could not be defined for the contiguous layers below the surface of these films to allow simulation of the surface characteristics in question. However, as the Sb-doped $\mathrm{SnO}_{2}$ films were grown on an intermediate UID $\mathrm{SnO}_{2}$ layer, the lattice mismatch was considerably decreased and the main sources of donors were the $\mathrm{Sb}$ dopants.

Homogeneous distribution of $\mathrm{Sb}$ atoms in the films, as confirmed by SIMS measurements, allowed for the calculation of the bulk Fermi level with respect to the CBM. The surface band bending and charge profile as a function of depth for Sb-doped $\mathrm{SnO}_{2}$ were obtained by simulation of the MottSchottky plots and space charge calculations. Downward band bending and surface electron accumulation were quantitatively demonstrated in $\mathrm{SnO}_{2}$ films. Both the surface band bending and the width of the accumulation region decrease with increasing $\mathrm{Sb}$ concentration. The unoccupied surface states become increasingly occupied, resulting in respective reduction of the space charge sheet density as the bulk Fermi level rises towards the CNL. Among the investigated samples, the largest band bending (unoccupied-surface-state density) was determined to be $-0.327 \mathrm{eV}\left(8 \times 10^{12} \mathrm{~cm}^{-2}\right)$ for a film with $\mathrm{Sb}$ and bulk electron concentration of $n_{\mathrm{Sb}}=9.8 \times 10^{17}$ and $n_{\mathrm{b}}=$ $1.1 \times 10^{18} \mathrm{~cm}^{-3}$, respectively.

\section{ACKNOWLEDGMENTS}

T.D.V. and D.O.S. acknowledge support from the Materials Design Network. T.D.V. acknowledges funding from the EPSRC under Grant No. EP/G004447/2. Wojciech Linhart 
is thanked for useful discussions. The work in TCD was supported by Science Foundation Ireland (PI Grants No. 06/IN.1/I92 and No. 06/IN.1/I92/EC07). Calculations at TCD were performed on the Lonsdale and Kelvin clusters as maintained by TCHPC, and the Stokes and Fionn clusters as maintained by ICHEC. The UCL/Diamond work presented here made use of the UCL Legion HPC Facility, the IRIDIS cluster provided by the EPSRC funded Centre for Innovation
(Grants No. EP/K000144/1 and No. EP/K000136/1), and the ARCHER supercomputer through support by the UK's HPC Materials Chemistry Consortium, which is funded by EPSRC Grant No. EP/L000202. The Science City Research Alliance (SCRA) facilities were used to collect the XPS spectra shown. The European Research and Development Agency (ERDF) is acknowledged for capital funding of the SCRA facility.
[1] V. Heine, Phys. Rev. 138, A1689 (1965).

[2] E. R. Leite, I. T. Weber, E. Longo, and J. A. Varela, Adv. Mater. 12, 965 (2000).

[3] M. Batzill, J. M. Burst, and U. Diebold, Thin Solid Films 484, 132 (2005).

[4] B. G. Lewis and D. C. Paine, MRS Bull. 25(8), 22 (2000).

[5] J. F. Wager, Science 300, 1245 (2003).

[6] L. F. J. Piper, T. D. Veal, M. J. Lowe, and C. F. McConville, Phys. Rev. B 73, 195321 (2006).

[7] I. Mahboob, T. D. Veal, C. F. McConville, H. Lu, and W. J. Schaff, Phys. Rev. Lett. 92, 036804 (2004).

[8] P. D. C. King, T. D. Veal, D. J. Payne, A. Bourlange, R. G. Egdell, and C. F. McConville, Phys. Rev. Lett. 101, 116808 (2008).

[9] K. H. L. Zhang, R. G. Egdell, F. Offi, S. Iacobucci, L. Petaccia, S. Gorovikov, and P. D. C. King, Phys. Rev. Lett. 110, 056803 (2013).

[10] L. F. J. Piper, L. Colakerol, P. D. C. King, A. Schleife, J. ZúñigaPérez, Per-Anders Glans, T. Learmonth, A. Federov, T. D. Veal, F. Fuchs, V. Muñoz-Sanjosé, F. Bechstedt, C. F. McConville, and K. E. Smith, Phys. Rev. B 78, 165127 (2008).

[11] A. Schleife, F. Fuchs, C. Rdl, J. Furthmller, and F. Bechstedt, Appl. Phys. Lett. 94, 012104 (2009).

[12] S. K. Vasheghani Farahani, T. D. Veal, P. D. C. King, J. ZúñigaPérez, V. Muñoz-Sanjosé, and C. F. McConville, J. Appl. Phys. 109, 073712 (2011).

[13] S. K. Vasheghani Farahani, V. Muñoz-Sanjosé, J. Zúñiga-Pérez, C. F. McConville, and T. D. Veal, Appl. Phys. Lett. 102, 022102 (2013).

[14] J. J. Mudd, T.-L. Lee, V. Muñoz-Sanjosé, J. Zúñiga-Pérez, D. J. Payne, R. G. Egdell, and C. F. McConville, Phys. Rev. B 89, 165305 (2014).

[15] D. Eger, A. Many, and Y. Goldstein, Surf. Sci. 58, 18 (1976).

[16] M. W. Allen, C. H. Swartz, T. H. Myers, T. D. Veal, C. F. McConville, and S. M. Durbin, Phys. Rev. B 81, 075211 (2010).

[17] T. Nagata, O. Bierwagen, M. E. White, M. Y. Tsai, Y. Yamashita, H. Yoshikawa, N. Ohashi, K. Kobayashi, T. Chikyow, and J. S. Speck, Appl. Phys. Lett. 98, 232107 (2011).

[18] O. Bierwagen, T. Nagata, M. E. White, Min-Ying Tsai, and J. S. Speck, J. Mater. Res. 27, 2232 (2012).

[19] K. T. Butler, J. Buckeridge, C. R. A. Catlow, and A. Walsh, Phys. Rev. B 89, 115320 (2014).

[20] O. Bierwagen, M. E. White, Min-Ying Tsai, T. Nagata, and J. S. Speck, Appl. Phys. Express 2, 106502 (2009).

[21] T. Nagata, O. Bierwagen, M. E. White, Min-Ying Tsai, and J. S. Speck, J. Appl. Phys. 107, 033707 (2010).

[22] P. Ágoston, K. Albe, R. M. Nieminen, and M. J. Puska, Phys. Rev. Lett. 103, 245501 (2009).
[23] P. Ágoston, C. Körber, A. Klein, M. J. Puska, R. M. Nieminen, and K. Albe, J. Appl. Phys. 108, 053511 (2010).

[24] A. Schleife, J. B. Varley, F. Fuchs, C. Rödl, F. Bechstedt, P. Rinke, A. Janotti, and C. G. Van de Walle, Phys. Rev. B 83, 035116 (2011)

[25] D. O. Scanlon, A. B. Kehoe, G. W. Watson, M. O. Jones, W. I. F. David, D. J. Payne, R. G. Egdell, P. P. Edwards, and A. Walsh, Phys. Rev. Lett. 107, 246402 (2011).

[26] M. Burbano, D. O. Scanlon, and G. W. Watson, J. Am. Chem. Soc. 133, 15065 (2011).

[27] D. O. Scanlon and G. W. Watson, Phys. Chem. Chem. Phys. 13, 9667 (2011).

[28] D. O. Scanlon and G. W. Watson, J. Mater. Chem. 22, 25236 (2012).

[29] D. O. Scanlon, Phys. Rev. B 87, 161201 (2013).

[30] D. O. Scanlon, J. Buckeridge, C. R. A. Catlow, and G. W. Watson, J. Mater. Chem. C 2, 3429 (2014).

[31] D. Waroquiers, A. Lherbier, A. Miglio, M. Stankovski, S. Poncé, M. J. T. Oliveira, M. Giantomassi, Gian-Marco Rignanese, and X. Gonze, Phys. Rev. B 87, 075121 (2013).

[32] M. E. White, M. Y. Tsai, F. Wu, and J. S. Speck, J. Vac. Sci. Technol. A 26, 1300 (2008).

[33] M. E. White, O. Bierwagen, M. Y. Tsai, and J. S. Speck, J. Appl. Phys. 106, 093704 (2009).

[34] G. Kresse and J. Hafner, Phys. Rev. B 49, 14251 (1994).

[35] G. Kresse and J. Furthmüller, Phys. Rev. B 54, 11169 (1996).

[36] J. P. Perdew, K. Burke, and M. Ernzerhof, Phys. Rev. Lett. 77, 3865 (1996).

[37] P. E. Blöchl, Phys. Rev. B 50, 17953 (1994).

[38] G. Kresse and D. Joubert, Phys. Rev. B 59, 1758 (1999).

[39] C. Adamo and V. Barone, J. Chem. Phys. 110, 6158 (1999).

[40] J. Paier, M. Marsman, K. Hummer, G. Kresse, I. C. Gerber, and J. G. Ángyán, J. Chem. Phys. 124, 154709 (2006).

[41] F. D. Murnaghan, Proc. Natl. Acad. Sci. USA 30, 244 (1944).

[42] G. Paasch and H. Übensee, Phys. Status Solidi B 113, 165 (1982).

[43] J.-P. Zöllner, H. Übensee, G. Paasch, T. Fiedler, and G. Gobsch, Phys. Status Solidi B 134, 837 (1986).

[44] H. Übensee, G. Paasch, and J.-P. Zöllner, Phys. Rev. B 39, 1955 (1989).

[45] S. K. Vasheghani Farahani, T. D. Veal, A. M. Sanchez, O. Bierwagen, M. E. White, S. Gorfman, P. A. Thomas, J. S Speck, and C. F. McConville, Phys. Rev. B 86, 245315 (2012).

[46] R. L. Petritz, Phys. Rev. 110, 1254 (1958).

[47] J. H. Scofield, J. Electron Spectrosc. 8, 129 (1976).

[48] J. J. Yeh and I. Lindau, At. Data Nucl. Data 32, 1 (1985).

[49] D. A. Shirley, Phys. Rev. B 5, 4709 (1972). 
[50] R. G. Egdell, J. Rebane, T. J. Walker, and D. S. L. Law, Phys. Rev. B 59, 1792 (1999).

[51] J. J. Mudd, T.-L. Lee, V. Muñoz-Sanjosé, J. Zúñiga-Pérez, D. Hesp, J. M. Kahk, D. J. Payne, R. G. Egdell, and C. F. McConville, Phys. Rev. B 89, 035203 (2014).

[52] L. Y. Lim, S. Lany, Y. J. Chang, E. Rotenberg, A. Zunger, and M. F. Toney, Phys. Rev. B 86, 235113 (2012).

[53] S. Lany, Phys. Rev. B 87, 085112 (2013).

[54] H. Peng, D. O. Scanlon, V. Stevanovic, J. Vidal, G. W. Watson, and S. Lany, Phys. Rev. B 88, 115201 (2013).

[55] D. Fröhlich, R. Kenklies, and R. Helbig, Phys. Rev. Lett. 41, 1750 (1978).
[56] K. Reimann and M. Steube, Solid State Commun. 105, 649 (1998).

[57] K. J. Button, C. G. Fonstad, and W. Dreybrodt, Phys. Rev. B 4, 4539 (1971).

[58] M. Feneberg, C. Lidig, K. Lange, M. E. White, M. Y. Tsai, J. S. Speck, O. Bierwagen, and R. Goldhahn, Phys. Status Solidi A 211, 82 (2014).

[59] Z. M. Jarzebski and J. P. Marton, J. Electrochem. Soc. 123, 333 (1976).

[60] P. D. C. King, T. D. Veal, P. H. Jefferson, S. A. Hatfield, L. F. J. Piper, C. F. McConville, F. Fuchs, J. Furthmüller, F. Bechstedt, H. Lu, and W. J. Schaff, Phys. Rev. B 77, 045316 (2008). 